\title{
Violencia: ética discursiva, representación social y saber
}

\author{
Violence: discourse ethics, social representation and knowledge \\ Violência: a ética do discurso, representação social e do conhecimento
}

DOI: http://dx.doi.org/10.23913/ricsh.v6i11.121

Mauricio Ávila Barba

Facultad de Filosofía, Universidad Autónoma de Querétaro, México

mauricio40@hotmail.com

\section{Resumen}

El presente trabajo analiza la noción de 'violencia' desde la perspectiva de las representaciones sociales (Serge Moscovici). En particular, indaga en las creencias de los individuos en torno a los saberes y las prácticas que pueden implementar para prevenirla, centrándose en la psicología, el sistema jurídico judicial, el arte y la religión. El objetivo es problematizar la discordancia o concordancia que puede haber en la manera como el sujeto se experimenta a sí mismo a partir de determinados saberes y prácticas (Michel Foucault), así como en otros horizontes hermenéuticos de comprensión del mundo; por ejemplo, su creencia en la asimetría entre la violencia y las estrategias de prevención que pueden programarse dentro de un proyecto de intervención comunitario.

Los resultados de este caso muestran que $87.4 \%$ de los estudiantes encuestados consideró que las causas de violencia tienen un origen psicológico: enojo, depresión, etcétera. Asimismo, la mayoría, $84.4 \%$, afirmó que la psicología es el saber más apropiado para prevenirla, por ejemplo, a través de medicación y terapia. De igual manera, $84.4 \%$ declaró que la falta de actividades artístico-culturales en su comunidad produce violencia, por lo que determinaron que la promoción de dichas actividades puede ayudar a prevenirla. Por otro lado, un porcentaje muy alto, $73.3 \%$, declaró que la religión no tiene ninguna relevancia en la prevención de la violencia; y $85 \%$ que no espera que se tomen medidas al respecto. A partir de estos resultados se concluye 
que existe concordancia entre los presupuestos de un proyecto artístico-cultural o psicológico y la representación social de los estudiantes encuestados, lo que quizás signifique mayor disposición de su parte a participar en un proyecto de vinculación. Por otro lado, un proyecto con aspectos religiosos puede ser recibido con dificultad por parte de los estudiantes. Estas circunstancias -encuentro y desencuentro, concordancia y discordancia (Jürgen Habermas)propician el entendimiento entre individuos con diferentes perspectivas del mundo, es decir, un encuentro entre sujetos con diferentes maneras de experimentarse a sí mismos, quienes no necesariamente llegan a un acuerdo.

Palabras clave: violencia, ética discursiva, representación social, saber, sujeto.

\section{Abstract}

On this paper, we discuss the notion of 'violence' under the siege of social representations (Serge Moscovici). Particularly, we inquire about the beliefs individuals have around knowledge and the practices that could be implemented to foresee and/or address violence, as well as that which could be expected to be done in the frame of such knowledge; we center our study on psychology, the judicial law system, art and religión. We use this case with the purpose of problematizing the discordance -or the concordance- that could exist between the ways in which an individual makes an experience of himself inside certain knowledge and practices (Michel Foucault) and other hermeneutic horizons of understanding of the world; for instance, the assymetry between that which the individuals of a community believe about violence and the strategies of attention and/or prevention of it that could be programmed in an intervention project in said community.

On the analized case, the results we obtained show that $87.4 \%$ of the students inquired, consider the causes of violence to be of a psychological carácter (anger, depresión, etcetera). Besides, $84.4 \%$ of the students sustained psychology as the most appropriate knowledge to prevent and/or attend violence, this medication and therapy. In a similar percentage, $84.4 \%$ of the inquired students declared that the lack of artistic and cultural activities, that could stimulate people creativity, in their communities, represent one of the causes of violence. As well, they judged that the promotion of artistic-cultural activities in their community would be one of the most appropiate médiums to prevent and attend violence. On the contrary, a very high percentage of students $(73.3 \%)$ declared that religion has no relevance on prevention or attention of 
violence; it isn't expected either that it is used to do anything about it (85\% of inquired students). From the results we conclude that there are a concordance between the premises of a culturalartistic project, or one that contemplates psychologicak intervention, and the social representation of violence held by the inquired students; condition that, maybe, means a better disposition to participate in a vinculation project that starts with the students. On the contrary, we infer that a vinculation project or an intervention that impplies religious aspects could confront many problems in its reception by the inquired students. Under these circumstances -between the encounter and disencounter, between a concordance and discordance-, with Jürgen Habermas, we propose some dialogical guidelines that enable the understanding between individuals that have dissimilar horizons of understanding of the world, the encounter between subjects that have different ways of making an experience of themselves -orientation that doesn't, necessarily, mean reaching an understanding among the parts.

Key words: violence, discourse ethics, social representation, knowledge, subject.

\section{Resumo}

Este artigo analisa a noção de 'violência' a partir da perspectiva das representações sociais (Serge Moscovici). Em particular, explora as crenças dos indivíduos sobre conhecimentos e práticas que podem ser implementadas para prevenir, concentrando-se em psicologia, sistema legal judicial, arte e religião. O objetivo é discutir o desacordo ou acordo que pode ser na forma como o sujeito experimenta-se de certos conhecimentos e práticas (Michel Foucault), bem como outros horizontes hermenêuticos de compreender o mundo; por exemplo, sua crença na assimetria entre as estratégias de prevenção da violência e que podem ser programados dentro de um projeto de intervenção comunitária.

Os resultados deste caso mostram que $87,4 \%$ dos estudantes pesquisados considerou que as causas da violência têm uma origem psicológica: raiva, depressão, e assim por diante. Além disso, a maioria, 84,4\% referiram que a psicologia é o mais apropriado para evitar, por exemplo, por meio de medicamentos e terapia conhecida. Da mesma forma, 84,4\% afirmaram que a falta de atividades artísticas e culturais em sua comunidade produz violência, então eles determinaram que a promoção de tais atividades podem ajudar a preveni-la. Por outro lado, uma percentagem 
muito elevada, 73,3\% disseram que a religião não tem nenhuma relevância na prevenção da violência; e $85 \%$ espera nenhuma ação foi tomada. A partir destes resultados conclui-se que há um acordo entre os orçamentos de um projeto de representação psicológica e social cultural ou artística dos alunos pesquisados, o que pode significar maior disposição de sua parte para participar de uma ligação projeto. Por outro lado, um projeto com aspectos religiosos pode ser recebido com dificuldade pelos alunos. Estas circunstâncias -Encuentro e desacordo, concordância e discordância (Jürgen Habermas) - promover o entendimento entre os indivíduos com diferentes perspectivas sobre o mundo, ou seja, um encontro entre indivíduos com diferentes maneiras de experimentar a si mesmos, que não necessariamente chegar a um acordo.

Palavras-chave: violência, ética do discurso, representação social, nomeadamente assunto.

Fecha Recepción: Junio 2016 Fecha Aceptación: Octubre 2016

\section{Introducción}

Es común que las personas confundan violencia con agresividad; ambas no se excluyen entre sí pero tampoco son equivalentes. El concepto de agresión se puede entender como "un estado motivacional, una característica de la personalidad, una respuesta a la frustración, un impulso intrínseco, o la satisfacción de un requerimiento de rol social aprendido" (Harré y Lamb, 1992, p. 27). Se puede abordar desde la perspectiva biológica (la agresión es natural en el hombre) o desde el aprendizaje social. Por ejemplo, Hogg y Vaugham (2011, p. 455) afirman que "los niños aprenden reglas que están a su alrededor, de modo que la agresión se internaliza”. De esa manera, los individuos aprenden a reaccionar de forma violenta ante distintas situaciones. Una diferencia entre agresión y violencia puede ser la siguiente: 
La agresividad sirve para definir el territorio de cada uno y hacer valer 'su derecho'. La violencia, en cambio, rompe los límites del propio territorio y los del otro, invade la relación y los vuelve confusos. Es una fuerza destructora de sí mismo y del otro. Es consensual definir al acto violento como todo atentado a la integridad física y psíquica del individuo, acompañado de un sentimiento de coerción y peligro (Perrone y Nannini, 1998, p. 30).

Diversas investigaciones han caracterizado a la 'violencia' y a la 'agresión' enfocándose principalmente en los condicionamientos sociales y/o biológicos que las causan y las mantienen, lo que puede establecer los mecanismos de prevención requeridos para disminuirlas, por ejemplo, el impulso de la educación artística, ética y cívica, el fortalecimiento de las condiciones materiales, del empleo, la seguridad social, la salud, etcétera. También destacan las ventajas y desventajas de los procedimientos correctivos: cárcel, leyes, hospitales psiquiátricos, etcétera (Gavira, Cuadrado y López, 2009, pp. 219-250; Hogg y Vaugham, 2011, pp. 451 - 453).

El presente trabajo tiene otro propósito. No busca hablar sobre las condiciones y las causas de la violencia, ni de las estrategias para su prevención y atención, sino indagar en las nociones de 'violencia' que son asumidas por los sujetos y los definen. Nos referimos a los saberes y a las prácticas, tales como la psicología, el sistema jurídico judicial, el arte y la religión. Indaga sobre los saberes que los sujetos creen que definen a la violencia, sobre sus causas y sobre lo que esperan de esos saberes. Un ejemplo: una persona puede tener la creencia de que la violencia es ocasionada por la miseria en la que los pobladores de su comunidad viven; por lo tanto, el mismo sujeto puede creer que la manera de solucionar el problema es a través de la creación de empleos, por lo que se requerirían ciertos profesionistas competentes para implementarlo, tal vez políticos, economistas, empresarios, etcétera.

La verdad es que el problema es más complejo. No se resuelve con un procedimiento mecanismo-causal que combate los efectos eliminando sus causas. Las causas de la violencia pueden ser múltiples y variadas; no siempre coinciden las estrategias de atención y de prevención con las disposiciones de los sujetos objeto de dichas estrategias. Por ejemplo, imaginemos el caso de un psicólogo social que realiza trabajo de campo en una comunidad donde los problemas los suele resolver el párroco; este psicólogo tiene un proyecto asistencial que implica una intervención terapéutica psicológica. ¿Cuál es el resultado de esta disimetría? No solamente el fracaso del proyecto, sino también una transgresión frontal de las formas de organización de la 
comunidad. Es muy probable que en un trabajo de campo o en un proyecto de intervención los psicólogos sociales, antropólogos, sociólogos, educadores sexuales, etcétera, se enfrenten a estas condiciones. ${ }^{1}$ Por esta razón, está justificado problematizar el encuentro entre diferentes formas de entender el mundo y, además, plantear algunas pautas dialógicas para un entendimiento. Para ejemplificar lo anterior en un caso particular, analizamos cómo se establece cierta simetría y asimetría entre las formas de concebir la violencia por parte de los individuos y las formas en que se cree -quizá por parte de instituciones gubernamentales, educativas, entre otras- se debería prevenir y atender. En otras palabras, discutir las formas en que los individuos se piensan -hacen experiencia de sí mismos- dentro de cierto saber sobre la violencia y, coyunturalmente, cómo esto puede empatar o no con las formas de prevención y de atención de la violencia. Esto lo analizamos con el objetivo de describir, por un lado, el encuentro entre individuos que tienen diferentes formas de entender la realidad y, por otro lado, junto con Jürgen Habermas (1999), de proponer algunas pautas dialógicas que posibiliten el encuentro entre dos distintos horizontes de comprensión del mundo.

Para lograr nuestro objetivo recurrimos a la noción de 'representación social' de Serge Moscovici, y a las de 'saber', 'sujeto' y 'subjetividad' de Michel Foucault. Además, echamos mano de un estudio de caso con la aplicación de encuestas a estudiantes del Bachillerato de la UAQ, Campus Amazcala, en el marco del proyecto de vinculación FoVin 2015, titulado "Teatro Náhuatl como recurso para reforzar los valores de los pobladores de Amazcala", realizado por Luz de Lourdes Álvarez, profesora de la Universidad Autónoma de Querétaro. El proyecto tiene el objetivo de colaborar en la atención y/o la prevención de la violencia, a través de la implementación de actividades creativas, como el teatro, entre los estudiantes de dicho campus. Cabe destacar que en este proyecto puede no haber una concordancia entre las formas que se están proponiendo para atender el problema de la violencia en la comunidad (el teatro, la intervención psicológica) y las formas en que los habitantes de esta comunidad conciben la

\footnotetext{
${ }^{1}$ Aunque la asimetría se podría plantear como un error metodológico que puede salvarse con un estudio previo al desarrollo de un proyecto de intervención, consideramos que vale la pena plantear el problema no solo como un asunto metodológico. Obviamente, también está el problema paradójico de si es lícito o no intervenir, desde un afán ilustrado y civilizador, en las formas de vida de las comunidades. Por el contrario, también está el problema de si es lícito no entrometerse y "dejar a su suerte" a las comunidades, por ejemplo, respetar sus formas de medicina para tratar enfermedades, aunque haya otros protocolos más efectivos en la medicina alópata.
} 
violencia (formas que podrían incluir, por ejemplo, la intervención del sacerdote, de la familia, entre otras). ${ }^{2}$

Nuestra intención principal es mostrar las formas de discordancia o de concordancia que hay entre los diferentes horizontes de comprensión del mundo, así como sugerir algunas pautas dialógicas ideales. Por esta razón, otro elemento más para lograr nuestro objetivo consiste en definir algunas pautas de la ética discursiva de Jürgen Habermas, como una vía de análisis de los problemas y de las posibilidades de entendimiento que surgen en el encuentro de dos horizontes de comprensión del mundo, en este caso la violencia. ${ }^{3}$

\section{ANTECEDENTES}

Partimos de la idea de que los saberes, ya sea sobre la violencia, la educación, la medicina, entre otros asuntos, implican y están acompañados, al menos, de prácticas concretas (tales como la posición de un profesor ante un estudiante, la relación médico-paciente, entre otras) y de instituciones (la escuela, la cárcel, el hospital, entre otras). Por ese motivo, un saber no nada más se puede explicitar para dar cuenta de su potencial explicativo o de su efectividad social, en otras palabras, en el caso de la violencia no solo podemos indagar sobre la verdad o la falsedad de los saberes religiosos, médicos, jurídicos, entre otros; o sobre la eficacia de las prácticas y de las instituciones que tienen como tarea prever y disminuir la violencia, sino también estudiarse como representaciones sociales.

Según Moscovici (1979, p. 18), la noción de 'representación social' refiere "un corpus organizado de conocimientos, gracias a las cuales los hombres hacen inteligible la realidad física y social, se integran en un grupo o en una relación cotidiana de intercambios, liberan los poderes de su imaginación”. Como señala G. N. Fishner (1990, p. 121), uno de los aspectos de las representaciones sociales a nivel funcional es el anclaje:

\footnotetext{
${ }^{2}$ Con lo anterior no estamos diciendo que de no haber concordancia se tiene que abandonar un proyecto de vinculación; por el contrario, de no haber concordancia tendría que replantearse y hacerse frente a las discordancias.

${ }^{3}$ Cabe destacar que el objetivo de este trabajo no se centra en mostrar lo que los estudiantes de dicho campus piensan sobre la violencia. Al contrario, nos interesa pensar la discordancia o la concordancia que resulta entre su percepción sobre la violencia y las formas con las que se cree se debe atender, por ejemplo, en el proyecto de teatro. Así, el asunto de este trabajo es de carácter epistémico-discursivo, en tanto se buscan con Habermas condiciones ideales de posibilidad de un diálogo entre diferentes horizontes de comprensión del mundo.
} 
La representación funciona como un sistema de interpretación; esto se traduce en el hecho de que se atribuirá a una representación un valor de utilidad social, así este sistema de interpretación se convierte en un sistema mediador capaz de regular la relación social, proponiendo repertorios, tipologías que servirán para evaluar los acontecimientos y las conductas.

Se propone que este sistema interpretativo, señalado por Fishner, puede componerse, entre otros elementos, por distintos saberes. Desde la perspectiva de Michel Foucault (2002, pp. 305-309), la noción de 'saber' no se refiere necesariamente al conocimiento científico; el saber implica las delimitaciones de y las relaciones entre: 1) Aquello de lo cual se puede hablar en una práctica discursiva: el dominio de los objetos; 2) El espacio donde el sujeto puede ubicarse para hablar de los objetos: posiciones subjetivas; 3) El campo de coordinación y de subordinación de los enunciados donde los conceptos aparecen, son definidos, se aplican y se transforman; y 4) Las posibilidades de utilización y de apropiación de los discursos.

En esta definición destacan los dos primeros aspectos: las prácticas discursivas y las posiciones subjetivas. La violencia se puede entender como un "dominio" que pertenece a diferentes saberes: la religión, el arte, la psicología, el derecho, entre otros; además, se puede tomar una posición en relación con estos saberes. Por ejemplo, en La historia de la sexualidad. Voluntad de saber, con relación a la noción de 'sexo reprimido', Foucault (2007, pp. 15 y 16) señaló que:

La pregunta que quiero formular no es ¿por qué hemos sido reprimidos?, sino ¿por qué decimos con tanta pasión, tanto rencor contra nuestro pasado más próximo, contra nuestro presente y contra nosotros mismos, que somos reprimidos?, ¿por cuál espiral hemos llegado a afirmar que el sexo es negado, a mostrar ostensiblemente que lo ocultamos, a decir que lo silenciamos, todo esto formulándolo con palabras explícitas, intentando que se lo vea en su más desnuda realidad, afirmándolo en la positividad de su poder y de sus efectos?

Con esto destaca una postura, una sujeción del sujeto a los discursos y a las prácticas de lo que se dice es la sexualidad. Cabe señalar que Foucault no está proponiendo que, una vez que sepamos que estamos sujetos a un discurso sobre lo sexual, podemos liberarnos de él y regresar a un estado de integridad o pulcritud. Simplemente menciona las condiciones bajo las cuales se 
definen los modos de subjetivación, esto es, la forma en que se llega a "ser sujeto legítimo de tal o cual tipo de conocimiento. "No es lo mismo cuando el conocimiento tiene la forma de exégesis de un texto sagrado, de una observación hecha por la historia natural o de un análisis del comportamiento de un enfermo mental. Son los procedimientos por los cuales el sujeto es llevado a observarse a sí mismo, a analizarse, a descifrarse, a reconocerse como dominio de un saber posible" (Foucault, 1994, p. 632). Así, siempre que se habla de un sujeto, éste es pensado como sujetado, al menos a un saber posible. Bajo estos supuestos, los saberes cuyo dominio es la violencia han definido al sujeto, quien de alguna forma queda sujetado a ellos, ya sea a través de la educación, de la familia, de la religión, por "decisión propia”, etcétera.

La noción de 'sujeto', equiparado aquí descuidadamente con la de 'individuo', puede considerarse dentro de una vertiente de sujeción y subjetivación; con ello Foucault quería decir que, frente a toda tradición cartesiana o fenomenológica que pondera un 'yo' sustancial, la noción de 'sujeto' debe definirse con base en el saber al que se encuentra sujetado. Por ejemplo, desde Foucault se puede definir al sujeto de un saber en tanto sujeto trabajador (economía), sujeto hablante (lingüística), sujeto viviente (biología); y también definirlo relacionándolo con prácticas divisorias como enfermo-sano (medicina, psiquiatría, etcétera), normal-delincuente (derecho); y, finalmente, definirlo sobre la base en la que los seres humanos orientan su vida: cuando hago experiencia de mí mismo como reprimido, como sano o como enfermo, como heterosexual, etcétera.

Que el sujeto se piense como sujetado no implica que esté condenado por las circunstancias y saberes que lo atraviesan. Al respecto, Foucault (1996, pp. 5 y 6) señaló:

Existe una tendencia que podríamos denominar, de una manera un tanto irónica, marxista académica o del marxismo académico, que consiste en buscar cómo las condiciones económicas de la existencia encuentran en la conciencia de los hombres su reflejo o expresión. Creo que esta forma de análisis tradicional en el marxismo universitario de Francia y de Europa en general tiene un defecto muy grave: el de suponer en el fondo que el sujeto humano, el sujeto de conocimiento, las mismas formas del conocimiento, se dan en cierto modo previa y definitivamente, y que las condiciones económicas, sociales y políticas de la existencia no hacen sino depositarse o imprimirse en este sujeto que se da de manera definitiva. 
Cuando hablamos de los saberes de las personas sobre la violencia, no solo nos referimos a aquellos que se autodenominan pre-científicos (saberes mágicos o religiosos), sino también a que en la actualidad y en algunas poblaciones "las ciencias inventan y proponen la mayoría de los objetos, conceptos, analogías y formas lógicas que usamos para encarar nuestras tareas económicas, políticas e intelectuales" (Moscovici, 1979, p. 13). Para explicar lo anterior, nos remitimos al análisis que este autor realizó en torno a la representación social y la difusión del psicoanálisis en la sociedad francesa de la segunda mitad del siglo XX (1979, p. 15):

En la medida que este saber (el psicoanálisis) ha dejado los espacios académicos para internarse en la vida de las personas, en la medida que los principios de su terapéutica son mejor conocidos, que sus conceptos son asimilados y discutidos, muchos individuos han comenzado a practicar un psicoanálisis salvaje sobre sí mismos y sobre los demás. Así, hablar de la sexualidad, de los conflictos con los padres, de tal o cual neurosis, se vuelve lícito y hasta recomendable.

En concreto, con el psicoanálisis -y lo mismo pasa con otras disciplinas, o con la religión, los mitos, etcétera-, desde la perspectiva de Moscovici, los sujetos "se hacen una opinión sobre su propia conducta o la conducta de su prójimo y actúan en consecuencia” (Moscovici, 1979, p. 12). A continuación, mencionamos tres ejemplos de cómo el psicoanálisis se ha convertido en un aspecto esencial de la experiencia de muchas personas, en el artículo "Siguiendo los pasos de Samuel Ramos: una propuesta metodológica de análisis de nuestras circunstancias" (Ávila, 2015, p. 282):

En una entrevista para la revista online Letra Urbana, el psicólogo Alejandro Dagfal advirtió que las costumbres y el lenguaje del argentino están fuertemente vinculados al psicoanálisis. Además, Dagfal señaló que el psicoanálisis en Argentina se vio bloqueado por los golpes militares de 1966 y 1976, por lo que solo pudo desarrollarse bajo regímenes democráticos (cfr. Dagfal, s/a). Por otro lado, la importancia del psicoanálisis en Argentina fue señalada también por el periódico de derecha Clarín. En este diario se advirtió la tendencia de los porteños a psicoanalizarse: "Mil pesos gasta por año, en promedio, una persona que va dos veces por semana a terapia, según estadísticas de la Asociación Psicoanalítica Argentina (APA). Hay 154 psicólogos por cada 100000 habitantes en la Argentina, cifra que representa el triple de la que hay en Estados Unidos" 
(2012). Consideramos entonces que este país sureño constituye un caso significativo, no solo para entender la institucionalización y el desarrollo del psicoanálisis, sino además para evidenciar condiciones de experiencia posible, al menos de los porteños.

Por otro lado, en su artículo "Variedades de un México freudiano", publicado el 01 de diciembre de 1979 en la revista Nexos, Carlos Monsiváis explica que por la noción de 'México freudiano' entiende "un orden de aceptación social de las realidades sexuales que deriva su plataforma básica del encuentro de ciertas clases en ascenso con algunas teorías de Freud".

Finalmente, en su libro Fenomenología del relajo, el filósofo mexicano Jorge Portilla (1984, p. 146) explicó lo que él consideraba crisis de Estados Unidos y advirtió:

Piénsese lo que se quiera del psicoanálisis como terapéutica..., [lo cierto es] que desde un punto de vista moral es o puede ser un sistema de excusas. Es obvio que a nivel de la psicología individual es una especie de otro yo, un Mrs. Hyde, que el psicoanálisis es capaz de domar por medio de una técnica adecuada. El yo es por una parte inocente de los desmanes del "ello", pero además estos desmanes pueden controlarse con una técnica racional. En su primer aspecto es indudablemente una excusa y permite asumir los impulsos descarriados en un horizonte de inocencia y en su segundo aspecto convierte el mal en algo dominable, en un fenómeno pasajero, por decirlo así artificial que no afecta el núcleo mismo de la personalidad, puesto que el "ello", a pesar de ser un modesto huésped en la psique, es concebido como radicalmente extraño, como reliquia eliminable, controlable, de un mundo infrahumano.

Los ejemplos anteriores muestran formas en las que ciertas perspectivas del psicoanálisis se han convertido en el "marco interpretativo" (Fishner) asumido por infinidad de individuos. A través de este saber, ven su mundo y actúan.

Como ya fue sugerido antes, un saber tiene implicaciones prácticas. Por ejemplo: si considero que la violencia es una consecuencia del desempleo, para poder preverla el paso más lógico sería la creación de empleos, la habilitación de los trabajadores o la implementación de toda estrategia que promueva el empleo. De igual forma, si creo que la violencia se ataca con la promoción de actividades artísticas, sería lógico promover proyectos de vinculación social en esta dirección. Sin embargo, el problema y su solución no siempre van de la mano. Veamos los siguientes casos 
en diversos contextos:

En los resultados de la Encuesta Nacional sobre Cultura Política y Culturas Ciudadanas (ENCUP), realizada en el año 2012 por la Secretaría de Gobernación (SEGOB), la cual tiene el objetivo de "obtener información sobre la cultura política y de las prácticas ciudadanas predominantes entre los mexicanos de 18 y más años" (SEGOB, 2012), se muestra una paradoja, una disimetría entre lo que se promueve, lo que se espera y lo que se quiere:

1. Seis de cada diez ciudadanos entrevistados ven en la democracia la forma de gobierno que más prefieren.

2. $65 \%$ de los ciudadanos entrevistados declaró tener poco interés en la política.

La paradoja consiste en que existe una población significativa en México que considera a la democracia como algo deseable, pero que no se interesa por participar en ella. ¿Cómo se puede conseguir la democracia con la no-participación ciudadana? ${ }^{4}$

La disimetría mencionada anteriormente nos hace pensar en otras. En su libro Multiculturalismo y Pluralismo, León Olivé analiza el conflicto zapatista, principalmente las diferencias lingüísticas y ontológicas que fueron un telón de fondo no explicitado en el "diálogo" entre las comunidades tojolabales y las autoridades federales de la república mexicana. Para mostrar el caso, Olivé se refiere a los estudios del lingüista Carlos Lenkersdorf, donde destaca:

Una sencilla oración como '(lo) dije, ustedes (lo) escucharon', no admite una traducción exacta al tojolabal. Esta oración se refiere a un hecho al que ninguna frase en tojolabal puede referirse. [El] hecho al que alude la frase en español es imposible de reconocer desde la visión del mundo de los tojolabales. Pero el hecho existe en virtud de la estructura misma de la lengua española. Así, no es que los tojolabales no la puedan reconocer, sino que eso no existe como hecho en su mundo (Olivé, 2007, p. 109).

\footnotetext{
${ }^{4}$ No es muy claro que la no-participación sea una opción democrática, pero eso no se discute ahora. También es necesario aclarar que la asimetría podría tener muchas causas, por ejemplo, la no-credibilidad en el sistema electoral, en los políticos, entre otras. Sin embargo, y pese a esto, creemos que es importante mencionar este caso porque nos permite ver los diferentes horizontes implicados en el análisis político en México.
} 
Olivé destacó que las diferencias lingüísticas no presentan simples problemas de traducción. Al contrario, con ello están en juego distintas formas de comprender, de experimentar y de interactuar con la realidad. Así, advierte que "las comunidades indígenas de Chiapas y los hispanohablantes de las sociedades modernas viven en mundos diferentes" (Olivé, 2007, p. 95). La paradoja que queremos destacar en este caso es que gran parte de la comunidad en conflicto estaba ordenada desde una forma de organización social, en la cual la solución de los conflictos no se formulaba por medio de un "acuerdo de la mayoría"; por el contrario, sin unanimidad no es posible el acuerdo (Olivé, 2007, p. 93). Es común pensar que la democracia significa un acuerdo entre la mayoría mediante el voto. Entonces la pregunta obligada es: ¿cómo se puede establecer un diálogo democrático bajo estas condiciones? Si no se contempla esta diferencia, la solución puede ser un tanto artificial y tendenciosa.

En la Encuesta Nacional sobre la Percepción Pública de la Ciencia y la Tecnología en México 2015 (ENPECYT), realizada por el Instituto Nacional de Estadística y Geografía (INEGI, 2015), en el rubro IV Comprensión y percepción de la ciencia y la tecnología, se hace un estudio entre las personas que creen en la ciencia o en la fe (religión), en ambas o en ninguna; y otro sobre el medio adecuado para el tratamiento de enfermedades que la ciencia no reconoce (acupuntura, quiropráctica, homeopatía, limpias). Este estudio se realizó en una población de 36068616 individuos (16 988488 hombres y 19080128 mujeres). Los resultados fueron los siguientes: 
Figura 1. Resultados de las encuestas (por cantidades)

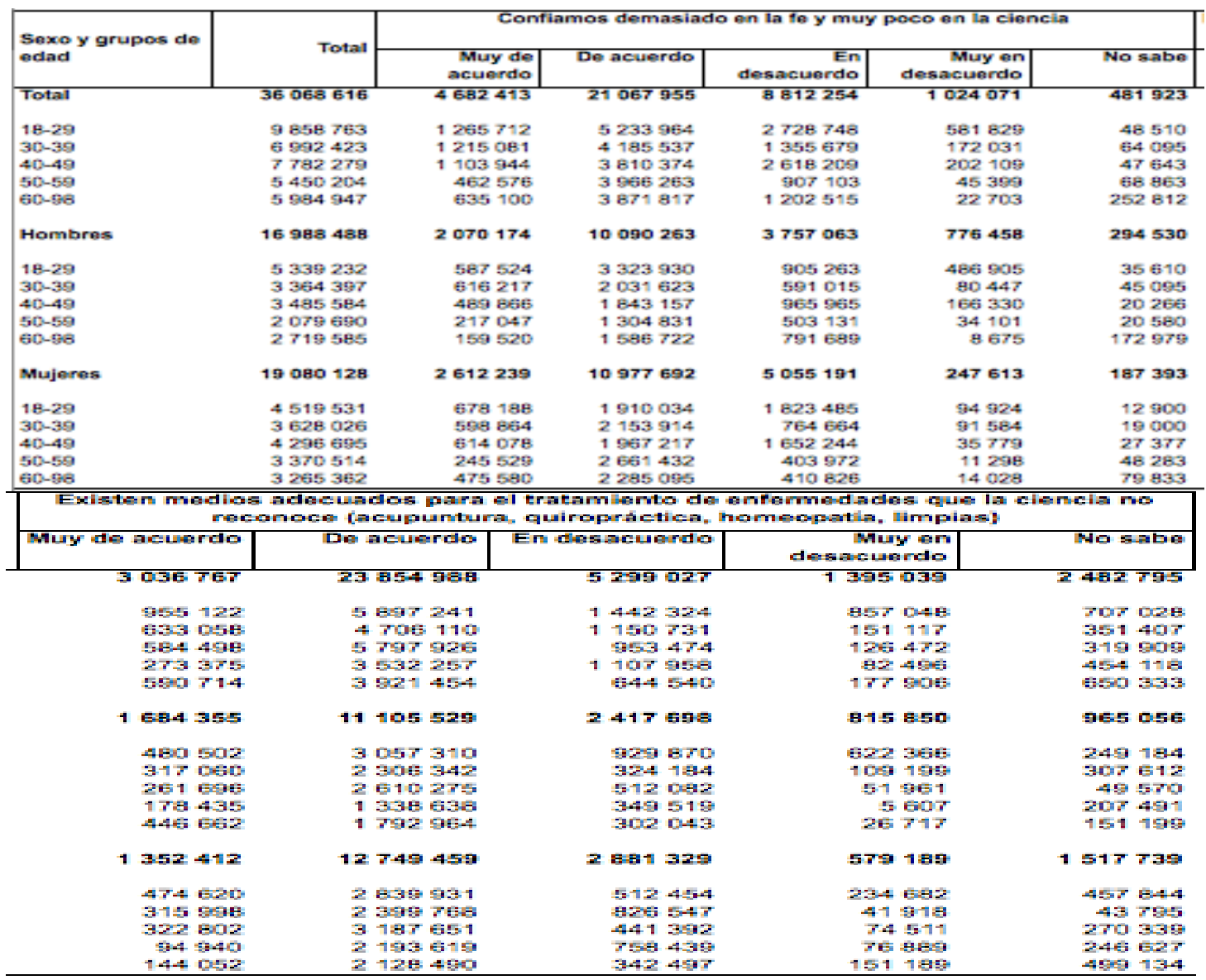

Fuente: INEGI, 2015 
Figura 2. Resultados encuestas (por porcentajes)

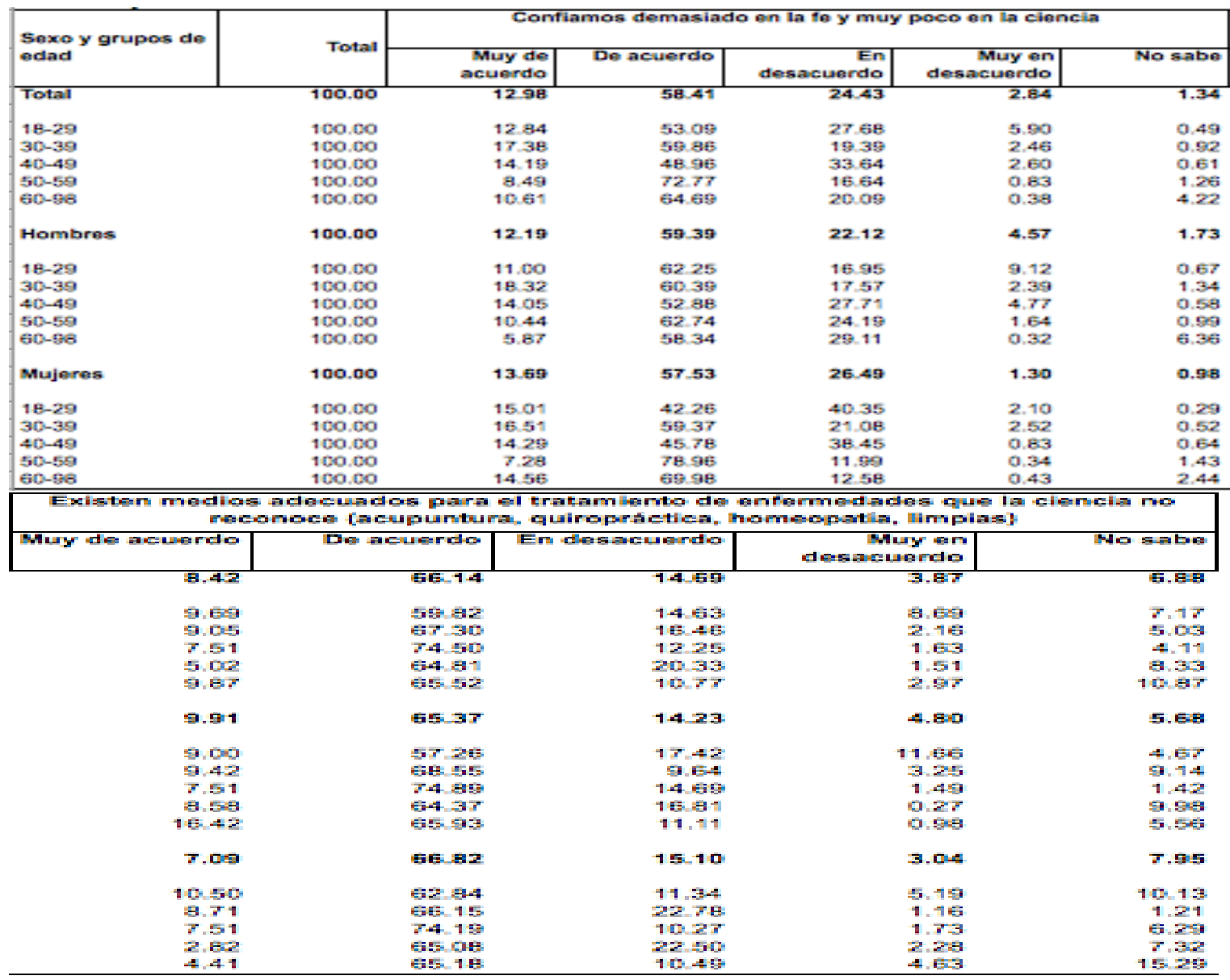

Fuente: INEGI, 2015.

No sorprende que haya un alto porcentaje de personas que creen más en la fe o en las limpias que en la ciencia. En México, lo anterior es predecible con cierta facilidad y certeza. Lo que llama la atención es si esto debería ser tomado en cuenta para políticas públicas, proyectos de intervención en comunidades, etcétera. Suponemos que sí. ${ }^{5}$

Los ejemplos arriba mencionados muestran las diferentes formas en que los sujetos se experimentan a sí mismos dentro del marco de ciertos saberes, como la psicología o el psicoanálisis. Además, muestran las asimetrías que puede haber, por ejemplo, en un diálogo democrático, en las formas de gobierno o en la atención de enfermedades. A continuación,

\footnotetext{
${ }^{5}$ En el estado de Querétaro existe un alto porcentaje de católicos: 89.3\% de la población, según el INEGI (2010). Por tanto, ¿podría proceder una iniciativa pública a favor del aborto en dicho Estado? Si en realidad se llevara la voz de los ciudadanos al Congreso, sería muy problemático y poco probable que fuera aprobada.
} 
planteamos este problema en un caso de intervención dentro del marco de un proyecto de vinculación.

\section{MÉTODO}

Para investigar en un caso concreto la concordancia y/o la discordancia señaladas anteriormente, se expone el siguiente estudio de caso, con el proyecto de vinculación "Teatro Náhuatl como recurso para reforzar los valores de los pobladores de Amazcala”, de la profesora Luz de Lourdes Álvarez. Este proyecto tiene el objetivo de ayudar, a través del teatro, a dar solución a los problemas de violencia. El objetivo es saber si las formas propuestas como mecanismo de intervención en el proyecto sobre violencia y teatro en la comunidad de Amazcala, Querétaro, son congruentes con los saberes que las personas de la comunidad consideran deben intervenir en la solución del problema de violencia; o si, por el contrario, se considera que no hay pertinencia del teatro o del arte. En este caso concreto, están programadas actividades lúdicas-creativas con la puesta en escena de una obra de teatro náhuatl.

Insistimos en que, aunque no haya concordancia entre los presupuestos del proyecto de teatro y las formas en que los estudiantes piensan la violencia, la propuesta no debe abortarse. Por el contrario, podrían buscarse pautas de entendimiento mutuo para aceptar o rechazar las propuestas del proyecto.

De acuerdo con la noción de 'saber' propuesta por Foucault, se delimitan las cuestiones planteadas en las encuestas: ${ }^{6}$

1. Las preferencias de los estudiantes por el saber que se debería encargar de prever y/o atender la violencia: arte (teatro), psicología, religión y sistema jurídico.

2. Lo que esperan los estudiantes que se haga para prevenir y/o atender la violencia, por ejemplo: que vuelva creativas a las personas violentas, que las aconseje y les dé valores espirituales, que las medique y/o les dé terapia, o que las castigue y las encierre.

A estos dos aspectos se suma el de la opinión que tienen los estudiantes sobre las causas de la violencia.

\footnotetext{
${ }^{6}$ No consideramos que los entrevistados tienen una teoría completa, coherente y justificada sobre la violencia. Apelamos a que suponen algo sobre la violencia de manera fragmentada, y que aun así esto determina su experiencia y perspectivas sobre ella.
} 
Así, en resumen, las encuestas nos ofrecen tres aspectos: 1. Las causas de la violencia, 2. Los saberes que deben atender la violencia y 3. Lo que se espera que se haga para prevenir y/o atender la violencia.

\section{SELECCIÓN DE MUESTRA}

Estudiantes del Bachillerato UAQ-Amazcala, en el municipio de "El Marqués”, en Querétaro.

Edad:15 a 19 años.

Muestra: 74 estudiantes.

Población: 90 estudiantes.

\section{FORMAS DE RECOLECCIÓN DE DATOS}

Encuesta. $^{7}$

\section{RESULTADOS}

Tabla 1. ¿Cuáles son las disciplinas, los saberes y las prácticas más adecuadas para prevenir y atender la violencia en mi comunidad?

\begin{tabular}{|c|c|c|c|c|c|c|}
\hline \multirow[t]{2}{*}{ Muestra/Sexo } & \multirow[t]{2}{*}{ Total } & \multicolumn{5}{|c|}{$\begin{array}{l}\text { Considero que la psicología es la más adecuada para prevenir y atender la violencia } \\
\text { en mi comunidad. }\end{array}$} \\
\hline & & $\begin{array}{l}\text { Muy de } \\
\text { acuerdo }\end{array}$ & De acuerdo & En desacuerdo & $\begin{array}{ll}\text { Muy en } \\
\text { desacuerdo }\end{array}$ & No sé \\
\hline Muestra & $76^{*}$ & 9 & 55 & 5 & 1 & 5 \\
\hline Hombres & 34 & 5 & 23 & 2 & 0 & 4 \\
\hline Mujeres & 39 & 3 & 31 & 3 & 1 & 1 \\
\hline $\begin{array}{l}\text { Personas que } \\
\text { no } \\
\text { identificaron }\end{array}$ & 3 & 2 & 1 & 0 & 0 & 0 \\
\hline
\end{tabular}

*Una persona no contestó este rubro. Por esta razón la muestra es de 76 estudiantes.

\begin{tabular}{|c|c|c|c|c|c|c|}
\hline \multirow[t]{2}{*}{ Muestra/Sexo } & \multirow[t]{2}{*}{ Total } & \multicolumn{5}{|c|}{$\begin{array}{l}\text { Considero que la religión es la más adecuada para prevenir y atender la violencia en } \\
\text { mi comunidad. }\end{array}$} \\
\hline & & $\begin{array}{ll}\begin{array}{l}\text { Muy } \\
\text { acuerdo }\end{array} & \text { de } \\
\end{array}$ & De acuerdo & En desacuerdo & $\begin{array}{ll}\begin{array}{l}\text { Muy } \\
\text { desacuerdo }\end{array} & \text { en } \\
\end{array}$ & No sé \\
\hline Muestra & 77 & 4 & 13 & 36 & 22 & 2 \\
\hline Hombres & 34 & 2 & 6 & 12 & 13 & 1 \\
\hline Mujeres & 40 & 2 & 6 & 22 & 9 & 1 \\
\hline $\begin{array}{l}\text { Personas que } \\
\text { no se } \\
\text { identificaron }\end{array}$ & 3 & 0 & 1 & 2 & 0 & 0 \\
\hline
\end{tabular}

\footnotetext{
${ }^{7}$ Realizamos dos precisiones: 1. En la encuesta se agregó una pregunta que hacía referencia a la autorización de los estudiantes para publicar los resultados de las encuestas. Todos dieron su autorización para publicar los resultados; y 2. Las encuestas fueron revisadas por la profesora Mariana Patricia Salinas Camberos y aplicadas por la profesora Luz de Lourdes Álvarez.
} 


\begin{tabular}{|l|l|l|l|l|l|l|}
\hline Muestra/Sexo & Total & \multicolumn{4}{l|}{$\begin{array}{l}\text { Considero que el arte (el teatro, la música, etcétera) es el más adecuado para } \\
\text { prevenir y atender la violencia en mi comunidad. }\end{array}$} \\
\cline { 3 - 8 } & & $\begin{array}{l}\text { Muy de } \\
\text { acuerdo }\end{array}$ & De acuerdo & En desacuerdo & $\begin{array}{l}\text { Muy } \\
\text { desacuerdo }\end{array}$ & No sé \\
\hline Muestra & 77 & 15 & 46 & 11 & 2 & 3 \\
\hline Hombres & 34 & 6 & 19 & 7 & 0 & 2 \\
\hline Mujeres que & 40 & 9 & 24 & 4 & 2 & 0 \\
\hline $\begin{array}{l}\text { Personas que } \\
\text { no se } \\
\text { identificaron }\end{array}$ & 3 & 0 & & 0 & 0 & \\
\hline
\end{tabular}

\begin{tabular}{|c|c|c|c|c|c|c|}
\hline \multirow[t]{2}{*}{ Muestra/Sexo } & \multirow[t]{2}{*}{ Total } & \multicolumn{5}{|c|}{$\begin{array}{l}\text { Considero que el sistema normativo judicial (código penal, policía, tribunales, } \\
\text { etcétera) es el más adecuado para prevenir y atender la violencia en mi comunidad. }\end{array}$} \\
\hline & & $\begin{array}{ll}\text { Muy de } \\
\text { acuerdo }\end{array}$ & De acuerdo & En desacuerdo & $\begin{array}{ll}\text { Muy en } \\
\text { desacuerdo }\end{array}$ & No sé \\
\hline Muestra & 77 & 4 & 31 & 24 & 6 & 12 \\
\hline Hombres & 34 & 2 & 14 & 12 & 1 & 5 \\
\hline Mujeres & 40 & 2 & 17 & 11 & 5 & 5 \\
\hline $\begin{array}{l}\text { Personas que } \\
\text { no se } \\
\text { identificaron }\end{array}$ & 3 & 0 & 0 & 1 & 0 & 2 \\
\hline
\end{tabular}

Fuente: elaboración propia para el estudio "Violencia: ética discursiva, representación social y saber".

Tabla 2. ¿Qué espero que se haga para prevenir y atender la violencia en mi comunidad?

\begin{tabular}{|c|c|c|c|c|c|c|}
\hline \multirow[t]{2}{*}{ Muestra/Sexo } & \multirow[t]{2}{*}{ Total } & \multicolumn{5}{|c|}{$\begin{array}{l}\text { Espero que los psicólogos mediquen y den terapia a las personas violentas para que } \\
\text { ellas puedan superar sus problemas. }\end{array}$} \\
\hline & & $\begin{array}{l}\text { Muy de } \\
\text { acuerdo }\end{array}$ & De acuerdo & En desacuerdo & $\begin{array}{ll}\text { Muy en } \\
\text { desacuerdo }\end{array}$ & No sé \\
\hline Muestra & 77 & 15 & 50 & 9 & 2 & 1 \\
\hline Hombres & 34 & 6 & 22 & 6 & 0 & 0 \\
\hline Mujeres & 40 & 6 & 28 & 3 & 2 & 1 \\
\hline $\begin{array}{lr}\text { Personas que } \\
\text { no } \\
\text { identificaron }\end{array}$ & 3 & 3 & 0 & 0 & 0 & 0 \\
\hline
\end{tabular}

\begin{tabular}{|c|c|c|c|c|c|c|}
\hline \multirow[t]{2}{*}{ Muestra/Sexo } & \multirow[t]{2}{*}{ Total } & \multicolumn{5}{|c|}{$\begin{array}{l}\text { Espero que los sacerdotes promuevan la fe en 'Dios' y den consejos a las personas } \\
\text { violentas, así ellas se arrepentirán y dejarán de ser violentas. }\end{array}$} \\
\hline & & $\begin{array}{ll}\text { Muy de } \\
\text { acuerdo }\end{array}$ & De acuerdo & En desacuerdo & $\begin{array}{ll}\text { Muy en } \\
\text { desacuerdo }\end{array}$ & No sé \\
\hline Muestra & 77 & 1 & 11 & 22 & 37 & 6 \\
\hline Hombres & 34 & 1 & 4 & 7 & 20 & 2 \\
\hline Mujeres & 40 & 0 & 5 & 15 & 17 & 3 \\
\hline $\begin{array}{lr}\text { Personas que } \\
\text { no } \\
\text { identificaron }\end{array}$ & 3 & 0 & 2 & 0 & 0 & 1 \\
\hline
\end{tabular}




\begin{tabular}{|l|l|l|l|l|l|l|}
\hline Muestra/Sexo & Total & \multicolumn{4}{l|}{$\begin{array}{l}\text { Espero que los artistas promuevan actividades que permitan a las personas violentas } \\
\text { orientar sus inquietudes y comportamientos hacia actividades creativas. }\end{array}$} \\
\cline { 3 - 7 } & & $\begin{array}{l}\text { Muy de } \\
\text { acuerdo }\end{array}$ & De acuerdo & En desacuerdo & $\begin{array}{l}\text { Muy } \\
\text { desacuerdo }\end{array}$ & No sé \\
\hline Muestra & 77 & 22 & 43 & 5 & 3 & 4 \\
\hline Hombres & 34 & 7 & 21 & 3 & 2 & 1 \\
\hline Mujeres que & 40 & 14 & 21 & 2 & 0 & 1 \\
\hline $\begin{array}{l}\text { Personas que } \\
\text { no se } \\
\text { identificaron }\end{array}$ & 3 & 1 & 1 & 0 & & 2 \\
\hline
\end{tabular}

\begin{tabular}{|c|c|c|c|c|c|c|}
\hline \multirow[t]{2}{*}{ Muestra/Sexo } & \multirow[t]{2}{*}{ Total } & \multicolumn{5}{|c|}{$\begin{array}{l}\text { Espero que la policía detenga a las personas violentas y las encierre en la cárcel. } \\
\text { Esto será un ejemplo para otras personas, así ellas dejarán de ser violentas. }\end{array}$} \\
\hline & & $\begin{array}{ll}\text { Muy de } \\
\text { acuerdo }\end{array}$ & De acuerdo & En desacuerdo & $\begin{array}{ll}\begin{array}{l}\text { Muy en } \\
\text { desacuerdo }\end{array} & \\
\end{array}$ & No sé \\
\hline Muestra & 77 & 10 & 34 & 24 & 5 & 4 \\
\hline Hombres & 34 & 6 & 14 & 12 & 1 & 1 \\
\hline Mujeres & 40 & 4 & 17 & 12 & 4 & 3 \\
\hline $\begin{array}{lr}\text { Personas que } \\
\text { no } \\
\text { identificaron }\end{array}$ & 3 & 0 & 3 & 0 & 0 & 0 \\
\hline
\end{tabular}

Fuente: elaboración propia para el estudio "Violencia: ética discursiva, representación social y saber".

Tabla 3. ¿Cuál es una de las causas de la violencia en mi comunidad?

\begin{tabular}{|c|c|c|c|c|c|c|}
\hline \multirow[t]{2}{*}{ Muestra/Sexo } & \multirow[t]{2}{*}{ Total } & \multicolumn{5}{|c|}{$\begin{array}{l}\text { Problemas de personalidad: las personas violentas están deprimidas, enojadas, } \\
\text { tristes, etcétera. }\end{array}$} \\
\hline & & $\begin{array}{l}\text { Muy de } \\
\text { acuerdo }\end{array}$ & De acuerdo & En desacuerdo & $\begin{array}{ll}\text { Muy en } \\
\text { desacuerdo }\end{array}$ & No sé \\
\hline Muestra & 77 & 22 & 44 & 1 & 1 & 9 \\
\hline Hombres & 34 & 4 & 22 & 1 & 1 & 6 \\
\hline Mujeres & 40 & 17 & 20 & 0 & 0 & 3 \\
\hline $\begin{array}{l}\text { Personas que } \\
\text { no se } \\
\text { identificaron }\end{array}$ & 3 & 1 & 2 & 0 & 0 & 0 \\
\hline
\end{tabular}

\begin{tabular}{|c|c|c|c|c|c|c|}
\hline \multirow[t]{2}{*}{ Muestra/Sexo } & \multirow[t]{2}{*}{ Total } & \multicolumn{5}{|c|}{ Las personas violentas se han alejado de Dios y de la fe. } \\
\hline & & $\begin{array}{ll}\begin{array}{l}\text { Muy } \\
\text { acuerdo }\end{array} & \text { de } \\
\end{array}$ & De acuerdo & En desacuerdo & $\begin{array}{ll}\begin{array}{l}\text { Muy } \\
\text { desacuerdo }\end{array} & \text { en } \\
\end{array}$ & No sé \\
\hline Muestra & 77 & 9 & 6 & 21 & 26 & 16 \\
\hline Hombres & 34 & 2 & 2 & 10 & 12 & 8 \\
\hline Mujeres & 40 & 5 & 4 & 10 & 14 & 7 \\
\hline $\begin{array}{l}\text { Personas que } \\
\text { no } \\
\text { identificaron }\end{array}$ & 3 & 1 & 0 & 1 & 0 & 1 \\
\hline
\end{tabular}

\begin{tabular}{|l|l|l|l|l|l|l|}
\hline Muestra/Sexo & Total & \multicolumn{3}{|l|}{$\begin{array}{l}\text { En la comunidad hay pocas actividades artístico-culturales que promuevan la } \\
\text { creatividad de las personas. }\end{array}$} \\
\cline { 3 - 8 } & & $\begin{array}{l}\text { Muy de } \\
\text { acuerdo }\end{array}$ & De acuerdo & En desacuerdo & $\begin{array}{l}\text { Muy } \\
\text { desacuerdo }\end{array}$ & No sé \\
\hline Muestra & 77 & 25 & 40 & 10 & 0 & 2 \\
\hline Hombres & 34 & 12 & 15 & 6 & 0 & 1 \\
\hline Mujeres que & 40 & 13 & 23 & 3 & 0 & 0 \\
\hline $\begin{array}{l}\text { Personas que } \\
\text { no se } \\
\text { identificaron }\end{array}$ & 3 & 0 & 2 & 1 & 0 & \\
\hline
\end{tabular}




\begin{tabular}{|l|l|l|l|l|l|l|}
\hline Muestra/Sexo & Total & \multicolumn{3}{l}{$\begin{array}{l}\text { Las autoridades (policías, jueces, etcétera) no tienen un sistema adecuado para } \\
\text { vigilar y castigar a las personas violentas de la comunidad. }\end{array}$} \\
\cline { 3 - 7 } & & $\begin{array}{l}\text { Muy de de } \\
\text { acuerdo }\end{array}$ & De acuerdo & En desacuerdo & $\begin{array}{l}\text { Muy desacuerdo } \\
\text { den }\end{array}$ & No sé \\
\hline Muestra & 77 & 20 & 37 & 12 & 3 & 5 \\
\hline Hombres & 34 & 6 & 18 & 6 & 1 & 3 \\
\hline Mujeres que & 40 & 13 & 18 & 5 & 2 & 0 \\
\hline $\begin{array}{l}\text { Personas qu } \\
\text { no se } \\
\text { identificaron }\end{array}$ & 1 & 1 & 1 & 0 & \\
\hline
\end{tabular}

Fuente: elaboración propia para el estudio "Violencia: ética discursiva, representación social y saber".

En resumen y en relación a las causas de la violencia, los resultados muestran que hay una tendencia a creer que son de carácter psicológico ( $87.5 \%$ de la muestra), o que se deben a la falta de actividades artístico-culturales $(84.4 \%)$ y al deficiente sistema judicial para vigilar y castigar a las personas violentas $(74 \%)$. Por el contrario, hay un bajo índice en la percepción sobre la influencia de la religión en las causas de la violencia (6.1\%).

Con respecto a los saberes y a las prácticas que podrían tener un papel importante en la prevención y atención de la violencia, los resultados muestran que la psicología (83.1 \% de la muestra) y el arte $(79.2 \%)$ se consideran pertinentes para esta tarea. Solo $45.5 \%$ de la muestra considera que el sistema judicial para vigilar y castigar es pertinente en la prevención y la atención de la violencia; por otro lado, 38.9 \% dijo que no y $19.4 \%$ dijo que no sabe. En religión hubo un porcentaje alto de estudiantes que afirmaron que no es pertinente para prevenir y atender la violencia $(75.3 \%)$.

Finalmente, en lo que se espera de las acciones para prevenir y atender la violencia, los resultados muestran que hay una tendencia a creer que la psicología -medicación y terapia- (84.4 $\%$ de la muestra) y el arte -actividades artísticas- (84.4\%) podrían ser útiles para esta tarea. En el sistema jurídico -la policía-, $57.1 \%$ de la muestra considera que tiene un papel importante en la prevención y atención de la violencia; por otro lado, 37.6.3 \% considera que no y $6 \%$ no sabe. Con respecto a la religión, $76.6 \%$ dice que no tiene un papel importante. 


\section{DISCUSIÓN}

Con base en los resultados se puede afirmar que sí existe concordancia entre los objetivos del programa de Teatro Náhuatl y la percepción que tienen los estudiantes sobre las causas, los saberes y las prácticas para prevenir o atender la violencia. En dicha concordancia se puede incluir a la psicología y en menor medida al sistema jurídico normativo. Finalmente, se puede afirmar que puede haber mayor discordancia entre un proyecto de intervención religiosa y la representación social sobre la violencia de los estudiantes encuestados.

¿Qué se puede hacer entonces con las discordancias? Como ya se mencionó, según la Encuesta Nacional sobre Cultura Política y Culturas Ciudadanas (ENCUP), las personas muestran poco interés por la política, a pesar de considerar que la democracia es el sistema político más deseable. Por tanto, no es preferible cambiar de régimen político e implementar, por ejemplo, una dictadura. Por otro lado, si en la Encuesta Nacional sobre la Percepción Pública de la Ciencia y la Tecnología en México 2015 (ENPECYT) se pone de manifiesto que un porcentaje alto de los encuestados prefiere algún medio para el tratamiento de enfermedades que la ciencia no reconoce, tales como la acupuntura, ${ }^{8}$ no significa que se deban programar actividades de acupuntura, ni que se lleven sacerdotes a una comunidad muy religiosa, etcétera, en un proyecto de intervención.

No bastan las buenas intenciones y realizar, por ejemplo, proyectos de vinculación o de intervención humanitarios; es necesario analizar las formas de compresión del mundo y determinar, al menos como trabajo propedéutico, las concordancias y las disonancias entre las diferentes representaciones sociales, en este caso la violencia. En su artículo "El reino de la hipercultura: cosmopolitismo y civilización occidental”, Guilles Lipovetsky (2011, pp. 62-69) examinó el horizonte planetario de los derechos humanos. Ahí destacó las tensiones que se presentan en esta universalización, pues mientras los derechos humanos pueden representar principios de una moralidad generalizable y deseable, por otro lado, los propios derechos humanos pueden verse como un imperialismo moral, principios de injerencia humanitaria, de intervención en países "inmorales" o como un proceso de individualización que disuelve de forma creciente la alteridad de las culturas. ${ }^{9}$

\footnotetext{
${ }^{8}$ Recordemos que de los 36068616 individuos de muestra, $74.56 \%$ está de acuerdo, $18.56 \%$ no está de acuerdo y $6.88 \%$ no sabe.

${ }^{9}$ Por ejemplo, actualmente hay una discusión importante sobre el imperialismo moral que se difunde a través de Fogarty International Center, asociación que entre otras cosas impulsa la creación de centros de bioética en
} 
Por tanto, se trata de reconocer la diversidad en las formas de comprensión del mundo, los saberes y las prácticas que atraviesan la experiencia de los individuos, e implementar estrategias dialógicas que permitan, en la medida de lo posible, la comprensión mutua de los participantes en un proyecto o, incluso, en un conflicto. Aunque reconocemos que el encuentro entre diferentes horizontes de comprensión del mundo puede implicar aspectos legales o económicos para su correcto planteamiento y resolución, creemos que al menos en estos casos en principio se deberían de buscar acuerdos; lo cual no implica que los participantes en un diálogo o, en este caso, en un proyecto, acepten o valoren lo mismo. Ello solo involucra un intento de poner en evidencia los presupuestos -las representaciones sociales- que estructuran el mundo de los participantes. Para tal efecto, podríamos pensar en unas pautas ideales que propone J. Habermas en su ética del discurso. ${ }^{10}$

Aunque la ética del discurso de Habermas refiere una forma racional para ponerse de acuerdo en contenidos morales -asunto que ahora no problematizamos-, las pautas que estructuran esta ética del discurso pueden servir para buscar otro tipo acuerdos y no solo en los asuntos morales. Con relación a la ética del discurso de Habermas, María José Guerra Palmero (2015) señala que para que exista un diálogo se debe contemplar una situación de ideal de habla, lo cual implica:

La igualdad de oportunidades para exponer los propios puntos de vista y la libertad para expresarse, junto con el reconocimiento de los otros como interlocutores válidos son las premisas de la situación ideal de habla:

[1] La misma oportunidad (para todos) de emplear actos de habla comunicativos, de suerte que en todo momento tengan la oportunidad tanto de abrir un discurso como de perpetuarlo mediante intervenciones y réplicas, preguntas y respuestas.

[2] Igual oportunidad de hacer interpretaciones, afirmaciones, recomendaciones, dar explicaciones y justificaciones y de problematizar, razonar o refutar las pretensiones de validez de ellas, de suerte que a la larga ningún prejuicio quede sustraído a la tematización y a la crítica.

[3] Que todos tengan iguales oportunidades de emplear actos de habla representativos, esto es, de expresar sus actitudes, sentimientos y deseos. Pues solo la recíproca sintonización de los espacios de expresión individual y la complementariedad en el juego de proximidad y distancia en los contextos de acción garantizan que los agentes, también,

diferentes países (Philpott-Jones, Gefenas, MacPherson, Strosberg y Hall, 2016). Esto no invalida la pertinencia de los derechos humanos, por ejemplo, en el marco jurídico de un país. Por el contrario, se quiere destacar el horizonte problemático en el que se promocionan y se pretenden institucionalizar los derechos humanos.

${ }^{10}$ Hay que aclarar que esto solo puede prosperar en un diálogo o en un proyecto donde los participantes tomen la palabra o tengan posibilidades de apropiarse de ella; es un límite de la propuesta de Habermas. Quizá dicha propuesta no funcionaría en un proyecto asistencial en el cual no se pida la opinión de los participantes y se les conduzca hacia lo que se considera su bien, independientemente de lo que opinen; tampoco funcionaría en una situación en la cual no haya la disposición ni las condiciones para dialogar. 
como participantes del discurso, sean veraces unos con otros y hagan transparente su naturaleza interna.

[4] Los hablantes agentes tendrán la misma oportunidad de actos de habla regulativos, es decir, de mandar y oponerse, de permitir y prohibir, de hacer y retirar promesas, de dar razón y exigirla. Pues solo la completa reciprocidad de expectativas de comportamiento, que excluye privilegios en el sentido de normas de acción y valoración que solo obliguen unilateralmente, puede garantizar que la equitativa distribución formal de las oportunidades de abrir una discusión y proseguirla, se emplee también fácticamente para dejar en suspenso las coacciones de la realidad y pasar al ámbito de comunicación exento de experiencia y descargado de acción que es el discurso (Guerra, 2015, pp. 86-88).

Estos lineamientos ideales colocan a los participantes -de un diálogo, un conflicto, un proyecto, etcétera- en una situación de igualdad de condiciones de participación. Faltaría agregar elementos que comprometan a las personas que participan en este encuentro. Habermas (1999, pp. 43-68) señala que se deberían buscar al menos cuatro aspectos que darían a un diálogo condiciones mínimas de racionalidad: 1. Que lo que expreso pueda ser entendible por los demás; 2. Que los hechos a los que refiere en un diálogo sean posibles, confiables; 3. Que los dialogantes sean sinceros y 4. Que los dialogantes no se dejen llevar solo por sus pasiones (moralidad).

Si se cumplen estas condiciones o al menos se buscan, entonces se puede avanzar en la comprensión de los encuentros y desencuentros que ocurren cuando diferentes horizontes de comprensión del mundo -llámense representaciones sociales- se tropiezan, ya sea en una disputa, en un proyecto de vinculación, etcétera. Al poner en evidencia nuestros presupuestos sobre cómo es el mundo a través de un diálogo entre iguales, si bien con ello no se garantiza la solución de un problema ni el buen desarrollo de un proyecto, se favorece la definición de una ruta heurística que permite acuerdos y desacuerdos, y con ello el reconocimiento de rutas alternativas de trabajo. 


\section{CONCLUSIONES}

La noción de 'violencia', vista como una representación social, puede definirse por los saberes y las prácticas que determinan la experiencia de los sujetos. Este horizonte hermenéutico, a través del cual regulamos nuestros vínculos sociales y hacemos experiencia de nosotros mismo, nos procuramos, nos observamos, nos evaluamos, etcétera, se pone en juego en cualquier diálogo, práctica, proyecto de intervención, etcétera. Ello no significa que la solución de problemas tales como la violencia coincida necesariamente con las creencias de las personas, es decir, si una persona cree que la violencia es ocasionada por un espíritu maligno no se llega a inferir que en un proyecto de vinculación se deban programar ceremonias espiritistas. Por el contrario, como vimos en los ejemplos mencionados, tanto en los antecedentes como en el proyecto de Teatro Náhuatl, un aspecto relevante en toda interacción -y en este caso en una intervención- es poner en evidencia las asimetrías que podrían resultar del encuentro entre diferentes formas de ver y valorar la violencia.

Las reglas dialógicas ideales propuestas por J. Habermas podrían servir de pautas heurísticas condición que regula nuestro entendimiento- en la discusión y en el establecimiento de acuerdos necesarios, en este caso, para la organización y la realización de actividades con la finalidad de prevenir y/o atender la violencia. Sin embargo, las indicaciones de Habermas tienen limitaciones, pero no necesariamente las hacen inútiles. Para nosotros está claro que solo se pueden aplicar en situaciones donde las personas que intervienen en un diálogo o en un proyecto quieran tomar la palabra y mantenerse en este ejercicio; por el contrario, si es un proyecto de intervención donde la opinión de los involucrados no está presente, ya sea porque se les niega o porque ellos mismos no la exteriorizan, difícilmente podrá hacerse algo con la propuesta de Habermas. 


\section{Bibliografía}

Ávila Barba, M. (2015). "Siguiendo los pasos de Samuel Ramos: una propuesta metodológica de análisis de nuestras circunstancias”. En Revista Valenciana, núm. 15, enero- junio, 2015, Universidad de Guanajuato, pp. 261-287.

Fisher, G. N. (1990). Psicología social. Conceptos fundamentales, Madrid: Narcea S. A. de ediciones.

Foucault, M. (1994). "Foucault” en: M. Foucault, Dits et Écrits (Ewald, F.; Defert, D. (Eds.)), t. IV, pp. 631-636, París: Gallimard.

Foucault, M. (1996). La verdad y sus formas jurídicas, Barcelona: Editorial Gedisa.

Foucault, M. (2002). Arqueología del saber, México: Siglo XXI Editores.

Foucault, M. (2007). La historia de la sexualidad. Voluntad de saber. México: FCE.

Gaviria Stewart, H. [et. al. ] (coord.). (2009). Introducción a la psicología social, Madrid: Sanz y Torres.

Guerra Palmero, M. J. (2015). Habermas. La apuesta por la democracia, Buenos Aires: Bonalletra Alcompas, S. L.

Habermas, J. (1999). Teoría de la acción comunicativa I. Racionalidad de la acción y racionalización social, Madrid: Taurus humanidades.

Harré, R. y Lamb, R. (1992). Diccionario de Psicología social y de la personalidad, Barcelona: Paidós.

Hogg, M. A. y Vaughan, G. M. (2011). Social Psychology, London: Pearson Education Limited.

Instituto Nacional de Estadística y Geografía (INEGI). (2010). "Religión". Obtenido el 10 de diciembre de 2016 de http://www.beta.inegi.org.mx/temas/religion/

Instituto Nacional de Estadística y Geografía (INEGI). (2015). "Encuesta sobre la Percepción Pública de la Ciencia y de la Tecnología en México (ENPECYT) 2015”. Obtenido el 10 de diciembre de 2016 de http://www.beta.inegi.org.mx/proyectos/enchogares/especiales/enpecyt/2015/default.html

Instituto Nacional de Estadística y Geografía (INEGI). (2016). "La Encuesta Nacional sobre Cultura Política y Prácticas Ciudadanas". Obtenido el 10 de septiembre de 2016 de http://www.inegi.org.mx/est/contenidos/proyectos/encuestas/hogares/especiales/encup/de fault.aspx

León, O. (2007). Multiculturalismo y Pluralismo, México: Paidós/UNAM. 
Lipovetsky, G. (2011). "El reino de la hipercultura: cosmopolitismo y civilización occidental". En El occidente globalizado. Un debate sobre la cultura planetaria. Barcelona: Editorial Anagrama S. A.

Monsiváis, C. (1979). "Variedades de un México freudiano", Revista Nexos. Obtenido el 25 de diciembre de 2016 de http://www.nexos.com.mx/?p=3252

Moscovici, S. (1979). El psicoanálisis, su imagen y su público, Buenos Aires: Editorial Huemul S. A.

Perrone, R. y Nannini M. (1998). Violencia y abusos sexuales en la familia, Buenos Aires: Paidós.

Philpott-Jones S., Gefenas E., Macpherson C.C., Strosberg M.A., Hall R.T. (2016). Fogarty and Charge of Moral Imperialism: A Response to Hellmann et al. Obtenido el 25 de febrero de 2017 de $\quad$ https://www.ncbi.nlm.nih.gov/pubmed/?term=PhilpottJones \%20S\%5BAuthor\%5D\&cauthor=true\&cauthor_uid $=26845618$

Portilla, J. (1984). Filosofía del relajo, México: FCE/CREA.

Secretaría de Gobernación (SEGOB). (2012). Encuesta Nacional sobre Cultura Política y Culturas Ciudadanas (ENCUP). Obtenido el 10 de diciembre de 2016 de http://www.encup.gob.mx/es/Encup/Principales_resultados_2012 